\title{
Discoveries of New Radio Stars
}

THE first surveys of the radio sky revealed many radio objects but none was found in the position of a star visible to the naked eye. These first radio sources were referred to as dark stars. The earliest identifications were made with optical nebulae-supernova remnants, ionized hydrogen clouds around young stars and eruptive or peculiar galaxies. Indeed a simple calculation readily shows that the blackbody radiation from the optical disk of a star, whether it is a very bright $\mathrm{O}$ or $\mathbf{B}$ star or a red giant of large diameter, would be far below the limits of detection. Stars were then dismissed as being of no interest to radio astronomy.

Many years later two theoretical papers showed that under favourable conditions radio emission might be detectable from certain types of star. Schatzman in 1958 pointed out that because the radio emission from the Sun at the time of a flare can increase by factors of $10^{6}$ or more above the quiet Sun value it should be possible to detect the radio emission from flare stars which erupt into even more violent flare activity than the Sun. Such radio burst emission was found by Lovell at Jodrell Bank and Slee and colleagues at Sydney.

The other prediction was made by Weyman and Chapman in 1965 who pointed out that certain red giant stars had absorption lines with blue shifted structure which indicated a flow of gas from the star. Weyman and Chapman argued that this gas would be hot and would form an extensive envelope around the red giant making it detectable at short radio wavelengths; it would be more difficult to see at longer wavelengths because this envelope would be optically thick. On page 37 of this issue of Nature Altenhoff and Wendker report the first detection of a red giant (Betelgeuse $=\alpha$-Orionis) using the $100 \mathrm{~m}$ radio telescope which has recently been built at the Max Planck Institute for Radio Astronomy at Bonn. Previous attempts to detect Betelgeuse have been unsuccessful or uncertain because of the poorer sensitivity of thie smaller telescopes or the low frequencies used. The Bonn results show that Betelgeuse gives a signal of $8+1$ milli flux units $\left(10^{-29} \mathrm{~W} \mathrm{~m}^{-2} \mathrm{~Hz}^{-1}\right)$ at a frequency of $10.7 \mathrm{GHz}$. This flux is consistent with emission from the corona of the star. It is also interesting that Betelgeuse is an infrared source, which implies that the outflowing gas condenses to form a circumstellar cloud of dust grains.

Another group of stars has recently been discovered by Wade and Hjellming using high sensitivity aperture synthesis techniques at the National Radio Astronomy Observatory in West Virginia. These are all eclipsing binaries in which the emitting star is of spectral type B. In all cases there is clear evidence for an outward streaming of gas from the surface of the star. The stars in this class are $\alpha$-Sco (Antares-the blue component of the double star system), $\beta$-Per (Algol), $\beta$-Lyr and the star associated with the X-ray source, Cyg X-1. These four sources have a quiet state flux at $8 \mathrm{GHz}$ of about 10 milli flux units, but show in addition marked irregular increases in brightness which are probably associated with the ejection of material from the star and consequent changes in the radio opacity.

The radio emission from another star, No. 349 in the
Mt Wilson Catalogue, is also described in this issue. MWC 349 is rather difficult to classify because it lies behind about 10 mag of absorption in the constellation Cygnus where it most likely belongs to the Cygnus OB2 association. It is an emission-line B star which shows gas emission from its surface and has many similarities to the eclipsing binary B stars described earlier. Infrared observations suggest that it is surrounded by a dust cloud. The first radio detection of MWC 349 was made by Braes, Habing and Schoenmaker (Nature, 240, $230 ; 1972$ ); the new observations reported on pages 37 and 38 by Altenhoff and Wendker at $10.7 \mathrm{GHz}$ and by Baldwin, Harris and Ryle at $5.0 \mathrm{GHz}$ (using the $5 \mathrm{~km}$ aperture synthesis telescope at Cambridge) give a much clearer picture of its nature. Its radio flux continues to rise towards higher frequencies indicating that it is radiating by free-free emission from an optically thick gas cloud with an electron temperature of $9,000 \pm 2,000 \mathrm{~K}$. The Cambridge radio astronomers were able to measure the angular diameter ( 2.4 arc $s$ ), and thereby estimated the mass of the emitting cloud to be $9 \times 10^{-3}$ solar masses. Baldwin et al. point to the similarity of the radio continuum properties of MWC 349 with those of the continuum source associated with the $\mathrm{OH}$ masering object W3 which is thought to be the HII region around a recently formed star still cmbedded within the parent gas cloud.

There is clearly a reawakening of interest in stars among radio astronomers who are being encouraged by the high sensitivity of the new breed of filled and unfilled aperture radio telescopes which make feasible the detection of weak emission from the circumstellar gas. The objects detected so far are a fascinating collection-red giants, blue emission line stars with P Cygni type line profiles, X-ray sources, and compact clouds of gas, molecules and dust around recently formed stars. Radio observations are likely to provide important information about the evolution of these objects.- R. D. D.

\section{DMN and Cervical Cancer}

THE finding of what might have been dimethylnitrosamine (DMN) in pooled human cervical and vaginal discharge matter by J. S. Harington and his colleagues (see page 49 of this issue of Nature) raises several matters that merit discussion. First, although the study involved the examination of vaginal fluid from a hundred African women, the fact that the specimens were pooled for the purposes of chemical analysis means that the report constitutes no more than a single unconfirmed observation. In spite of the care taken in the collection of specimens the possibility of contamination of one or more of them cannot be ruled out, and even if it could, one is not to know whether the DMN allegedly found in the pooled material came from several or only one woman. Nor can it be assumed that DMN found in vaginal fluid is the result of synthesis by microbial action in the vagina. For one reason or another various objects and chemical preparations find their way into the vagina so that the possibility of 